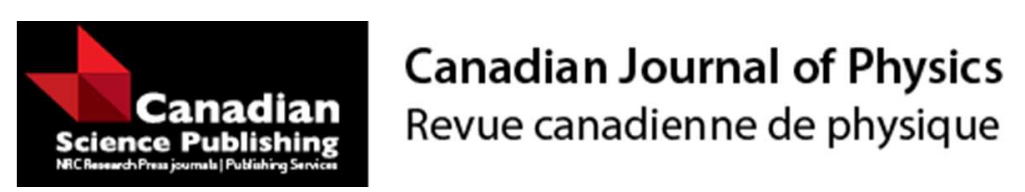

\title{
Analyses of alpha-alpha elastic scattering data in the energy range 53.4-119.9 MeV
}

\begin{tabular}{|r|l|}
\hline Journal: & Canadian Journal of Physics \\
\hline Manuscript ID & cjp-2015-0316.R3 \\
\hline Manuscript Type: & Article \\
\hline Date Submitted by the Author: & 05-Sep-2015 \\
\hline Complete List of Authors: & Shehadeh, Z.F.; Taif University, Physics \\
\hline Keyword: & $\begin{array}{l}\text { Alpha-alpha potential, Elastic scattering, Inverse scattering theory, } \\
\text { Identical particles, Schrödinger equation }\end{array}$ \\
\hline \multicolumn{2}{|l}{} \\
\hline
\end{tabular}

SCHOLARONE $^{\text {Ix }}$
Manuscripts 


\title{
Analyses of alpha-alpha elastic scattering data in the energy range 53.4-119.9 MeV
}

\author{
Z. F. Shehadeh \\ Physics Department, Faculty of Science, Taif University, Taif, Saudi Arabia \\ E-mail: zfs07@hotmail.com
}

\begin{abstract}
The differential and reaction cross sections for alpha-alpha elastic scattering at energies ranging from $50 \mathrm{MeV}$ to $120 \mathrm{MeV}$ (lab. system) have been clearly explained for the first time, by using a new optical potential type. This potential, which is different from all other proposed potentials, is composed of two real parts: one is an attractive squared Woods-Saxon and the other is a repulsive core of the WoodsSaxon form in addition to a surface Woods-Saxon form for the imaginary part. The nature of the real part has been determined from available phase shifts through using inverse scattering theory for the identical particles at a fixed energy, adopting the framework of Schrödinger equation. It is found that the repulsive real part is essential for improving the fit to the measured elastic differential cross sections, and in explaining the kink that appears at $r<1.0 \mathrm{fm}$ in the shape of the real part of the potential. Using this new potential, our calculated reaction cross sections are in reasonable agreement with the ones reported by both Darriulat et al. and Brown and Tang.
\end{abstract}

PACS numbers: 24.10.Ht, 25.10.+s, 25.55.Ci, 27.10.+h

Keywords: Alpha-alpha potential, Elastic scattering, Inverse scattering theory, Identical particles, Schrödinger equation 


\section{Introduction}

The importance of alpha particle in nuclear physics has been well established. This is mainly due to its unique properties summarized as : 1) It has zero spin and isospin. 2) It also has a high binding energy of $28.3 \mathrm{MeV}$, and a small root-mean-square radius of 1.44 fm. 3 ) It also plays a role in forming light cluster nuclei which contain alpha-particle substructures. The knowledge of these properties is necessary to understand $\alpha$-nucleus interaction and, as a prerequisite, the $\alpha-\alpha$ interaction.

As such, the determination of $\alpha-\alpha$ potential has been the subject of many experimental and theoretical studies. Below $50 \mathrm{MeV}$, Abdullah et al. [1] have recently suggested an $\ell$-independent non-monotonic real potential up to $34.2 \mathrm{MeV}$ (lab), where no inelastic channels are open, and a complex potential with non-monotonic real part from 35.1 to $47.3 \mathrm{MeV}$ (lab) to explain the available measured elastic angular distributions. This potential, which relies on the energy density functional theory with the sudden approximation, shows a remarkable success in explaining all the available data of energies reported therein.

On the other hand, Farid [2] has analyzed the ${ }^{4} \mathrm{He}-{ }^{4} \mathrm{He}$ elastic differential cross sections at energies ranging from 100 to $280 \mathrm{MeV}$ within the framework of the double-folding optical model. Chauhan and Khan [3],over the same energy range 100-280MeV, have analyzed the alpha-alpha elastic differential cross sections within the frame work of the Coulomb-modified Glauber model for nucleus-nucleus collisions. In analyzing their data for alpha-alpha elastic scattering at 280 and $620 \mathrm{MeV}$, Rao et al. [4] have concluded the failure of using only WoodsSaxon potential in reproducing the large angle data; and emphasized the need for adding a Woods-Saxon squared term to the real central potential. In a previous study, Nadasen et al. [5] have measured ${ }^{4} \mathrm{He}-{ }^{4} \mathrm{He}$ elastic differential cross sections at $158.2 \mathrm{MeV}$, and have analyzed the data within the framework of an optical-model potential. Also, Coker and Tamura [6] have made a phenomenological analysis of alphaalpha elastic scattering data [7] at 650 and $850 \mathrm{MeV}$. They have stressed the necessity for a short-range repulsive term added to the attractive real part of the potential.

In a more previous study, Darriulat et al. [8] have measured and analyzed the alpha-alpha elastic scattering data at seven energies between 53 and $120 \mathrm{MeV}$ (lab); namely, 53.4, 58.50, 63.9, 69.9 and 77.6, 99.6 and 119.9 MeV. In analyzing the data, they have used an $\ell-$ dependent potential terms composed of two real, attractive and repulsive, besides one imaginary of the Woods-Saxon type. The repulsive term of the real part is a short-radius repulsive core. The data at 99.6 and $119.9 \mathrm{MeV}$ have been reanalyzed by different workers [2, 3] using 
different potentials. Although all analyses have shown a good fit to the measured differential cross sections, they differ in accounting for the reaction cross sections. More clearly, although the calculated reaction cross sections by Farid [2] and Chauhan and Khan [3] have underestimated the reported values by Darriulat et al. by a factor of about 2 at the two energies (100 and $120 \mathrm{MeV})$ in common, our calculated values agree reasonably well with both Darriulat et al.'s [8] and Brown and Tang's values [9]. In addition, all proposed potentials and theoretical models $[2-6,8,9-16]$ did not only differ in the degree of their successes in explaining the measured angular distributions, but also disagree on the nature of the real part of the potential. This also has been emphasized by Jain and Joshi [17] who used $\alpha-\alpha$ phenomenological optical potentials at the laboratory bombarding energies from 77 to $200 \mathrm{MeV}$ in order to evaluate the $\alpha-\alpha$ transitional operator-effective interaction. The potentials used are the $\ell$ dependent $\alpha-\alpha$ optical potentials with repulsive core and the $\ell$ independent fully attractive ones. Unfortunately, all these studies did not decide precisely on the nature of the real part of the potential. On the other hand, and to the best of our knowledge, no other study tried to explain both differential and integral data at laboratory bombarding energies of 53.4, 58.50, 63.9, 69.9 and $77.6 \mathrm{MeV}$.

Previously, an inverse scattering theory (IST) at a fixed energy was used by Alam and Malik to determine the correct potentials required for a successful explanation of the experimental angular distributions for the scattering of two non-identical [18] besides identical particles [19] where reliable and suitable phase shifts are available. As such, and since the phase shifts are available for $\alpha-\alpha$ scattering cases [8] at the seven energies under consideration, we are motivated to use the IST to: a) settle the debate about the nature of the $\alpha-\alpha$ potential b) explain the long-standing unexplained $\alpha-\alpha$ elastic angular distribution data at 53.4, 58.50, 63.9, 69.9 and 77.6 MeV, using an $\ell$ independent optical potential c) confirm the capability and strength of the IST, used as a guide, in predicting the correct potentials.

In the following section, the theory concerning both the nature of alpha-alpha potential and IST for identical particles is presented. Results and discussions are included in Sect. 3. Finally, Sect. 4 presents the conclusions. 


\section{Theory}

\subsection{The nature of alpha-alpha potential}

The adopted optical potential, composed of three standard potential terms, has the following analytical form:

$$
V(r)=\frac{V_{o}}{\left[1+\exp \left(\frac{r-R_{o}}{a_{o}}\right)\right]^{2}}+\frac{V_{1}}{1+\exp \left(\frac{r-R_{1}}{a_{1}}\right)}+i\left(4 W_{s}\right) \frac{\exp \left(\frac{r-R_{i}}{a_{i}}\right)}{\left[1+\exp \left(\frac{r-R_{i}}{a_{i}}\right)\right]^{2}}
$$

with the Coulomb potential term, $V_{C}(r)$, is taken for a uniformly charged insulating sphere :

$$
V_{C}(r)= \begin{cases}\frac{Z_{1} Z_{2} e^{2}}{2 R_{C}}\left(3-\frac{r^{2}}{R_{C}^{2}}\right) & r \leq R_{C} \\ \frac{Z_{1} Z_{2} e^{2}}{r} & r>R_{C}\end{cases}
$$

where $Z_{1}=Z_{2}=2$ are the atomic numbers of the two colliding $\alpha$-particles. $e^{2} \cong 1.44 \mathrm{MeV} . f m$ and $R_{c}=2\left[1.3(4)^{1 / 3}\right]=2[2.0363]=4.07 \mathrm{fm}$ are the square of the electronic charge and the effective Coulomb radius, respectively.

In Eq. (1), $V(r)$ consists of a real part composed of an attractive squared Woods-Saxon and a repulsive Woods-Saxon terms, and an imaginary phenomenological attractive surface Woods-Saxon term, respectively. It is simply Farid's potential supplemented by Darriulat's repulsive Woods-Saxon core. To have an idea about the nature of the real part of the potential, the IST [18] for identical particles has been used, as a guide, in extracting the real potential points from the available phase shifts. This theory is summarized in the following subsection. 
Using the inverted potential points, the parameters of the real part of the potential given in (1) were adjusted to keep the analytical form of the potential very close to the inverted potential points in conjunction with producing a good fit to the measured cross sections.

The spherical symmetric potential, $V(r)$, is inserted in the radial part of Schrödinger's equation for the $\ell$ th partial wave given by

$$
\left[d^{2} / d r^{2}+k^{2}-U(r)+U_{C}(r)-\frac{\ell(\ell+1)}{r^{2}}\right] \psi_{\ell}(r)=0
$$

In Eq. (3), $k^{2}, U(r)$ and $U_{C}(r)$ are given by

$$
\begin{aligned}
& k^{2}=\frac{2 \mu}{\hbar^{2}} E \\
& U(r)=\frac{2 \mu}{\hbar^{2}} V(r) \\
& U_{C}(r)=\frac{2 \mu}{\hbar^{2}} V_{C}(r)
\end{aligned}
$$

where $\mu$ and $E$ are the reduced mass and the center of mass energy. Equation (3) should also satisfy the boundary condition

$$
\lim _{r \rightarrow 0} \psi_{\ell}(r) \approx(k r)^{\ell+1}
$$

The inner solution, for $r<R$, is obtained by solving Eq. (3) numerically. For the scattering of charged particles as in our case, the outer solution, for $r \geq R$, is the well-known regular, $F_{\ell}$, and irregular, $G_{\ell}$, Coulomb wave functions expressed as

$$
\psi_{\ell}(r)=F_{\ell}(\gamma, k r)+T_{\ell}\left[G_{\ell}(\gamma, k r)+i F_{\ell}(\gamma, k r)\right]
$$

where the matrix $T_{\ell}$ is related to the phase shift $\delta_{\ell}$, which is complex, by:

$$
T_{\ell}=\frac{e^{2 i \delta_{\ell}}-1}{2 i}
$$

and $\gamma$ is the Sommerfeld parameter given by : 


$$
\gamma=\frac{\mu Z_{1} Z_{2} e^{2}}{\hbar^{2} k}
$$

To determine $\delta_{\ell}, \mathrm{s}$, the logarithmic derivatives of the two solutions, the outer and the inner ones, are matched at the surface of the nucleus, i.e. at $r=R$. As such, one can calculate the total scattering amplitude at an angle $\theta$ in the center of mass system, $f(\theta)$. For the scattering of two identical particles,

$$
f(\theta)=f_{c c}(\theta)+\frac{1}{2 i k} \sum_{\ell=0}^{\infty}(2 \ell+1) \exp \left(2 i \sigma_{\ell}\right)\left[\exp \left(2 i \delta_{\ell}\right)-1\right]\left[P_{\ell}(\cos \theta)+P_{\ell}(\cos (\pi-\theta))\right]
$$

Since $\cos (\pi-\theta)=-\cos \theta$ and $P_{\ell}(-\cos \theta)=(-1)^{\ell} P_{\ell}(\cos \theta)$, equation (11) reads

$$
f(\theta)=f_{c c}(\theta)+\frac{1}{2 i k} \sum_{\ell=0}^{\infty}(2 \ell+1) \exp \left(2 i \sigma_{\ell}\right)\left[\exp \left(2 i \delta_{\ell}\right)-1\right]\left[P_{\ell}(\cos \theta)+(-1)^{\ell} P_{\ell}(\cos \theta)\right]
$$

The Coulomb scattering amplitude, $f_{c c}(\theta)$, is also expressed as

$$
f_{c c}(\theta)=f_{c}(\theta)+f_{c}(\pi-\theta)
$$

where the Coulomb scattering amplitude, $f_{c}(\theta)$, and the Coulomb phase shift, $\sigma_{\ell}$, are given by

$$
\begin{aligned}
& f_{c}(\theta)=\frac{-\gamma}{2 k \sin ^{2}(\theta / 2)} \exp \left[-i \gamma \ln \left\{\sin ^{2}(\theta / 2)\right\}+2 i \sigma_{0}\right] \\
& \sigma_{\ell}=\arg \Gamma(\ell+1+i \gamma)
\end{aligned}
$$

For identical particles, $Z_{1}=Z_{2}=Z$ and $\gamma$ in (10) becomes $\gamma=\mu(Z e)^{2} / \hbar^{2} k=(Z e)^{2} / \hbar v$ where $v$ is the relative velocity of the projectile and the target.

Again knowing $\delta_{\ell}$, the elastic differential cross section, $\frac{d \sigma}{d \Omega}$, and the total cross section, $\sigma_{r}$, can be calculated:

$$
\frac{d \sigma}{d \Omega}=|f(\theta)|^{2}
$$




$$
\sigma_{r}=\frac{2 \pi}{k^{2}} \sum_{\ell=0}^{\infty}(2 \ell+1)\left[1-\left|S_{\ell}\right|^{2}\right]
$$

where $S_{\ell}=e^{2 i \delta_{\ell}}$ is the S-matrix.

From Eq. (11) it is clear that only even partial waves contribute to the scattering amplitude. It should also be emphasized that the upper limit of the sum is restricted by the classical limit of the angular momentum which actually does not go to infinity.

\subsection{Inverse scattering theory for identical particles}

Now it is well-known that the inverse scattering method, used as a guide, proves to be very successful in predicting the nature of the potential in the scattering problem. Since the method has been remarkably successful in determining the nuclear potential for non-relativistic scattering of two non-identical particles [18] as well as identical particles [19] within the framework of Schrödinger equation, the method has been recently developed by Shehadeh and Malik to treat relativistic scattering cases; namely $\pi^{ \pm}$- nucleus elastic scattering, within the framework of Klein-Gordon equation [20-26]. Taking all this into consideration besides the extra test for the IST in correct guidance, the parameterization of the potential are going to be applied by us to the method for the scattering of two alpha particles over the energy range 50-120 MeV (lab) where phase shift analyses are available [8]. As mentioned in the above subsection, the method has been outlined previously [18] and it is reformulated and repeated here for emphasis.

Considering the nuclear potential only, equation (3) reads

$$
\left[d^{2} / d r^{2}+k^{2}-U(r)-\frac{\ell(\ell+1)}{r^{2}}\right] \psi_{\ell}(r)=0
$$

Defining the function $\varphi_{\ell}(r)$ as 


$$
\varphi_{\ell}(r)=(k r)^{-(\ell / 2+1)} \psi_{\ell}(r)
$$

equation (18) becomes

$$
\left[\frac{d^{2}}{d r^{2}}+\frac{\ell+2}{r} \frac{d}{d r}+k^{2}-U(r)-\frac{\ell(3 \ell+2)}{4 r^{2}}\right] \varphi_{\ell}(r)=0
$$

From (7) and (19), it is clear that $\varphi_{\ell}(r)$ satisfies the following boundary condition:

$$
\lim _{r \rightarrow 0} \varphi_{\ell}(r) \approx(k r)^{\ell / 2}
$$

Converting the differential equation, (20), into a different equation, one gets

$$
\varphi_{n+1}=A_{n}(\ell) B_{n}(\ell) \varphi_{n}+C_{n}(\ell) \varphi_{n-1} \quad n=1,2, \ldots, N
$$

with the following definitions

$$
\begin{aligned}
& A_{n}(\ell)=2-\Delta^{2} k^{2}+\Delta^{2} U_{n}+\frac{\ell(3 \ell+2)}{4 n^{2}} \\
& B_{n}(\ell)=\frac{2 n}{\ell+2+2 n} \\
& C_{n}(\ell)=\frac{\ell+2-2 n}{\ell+2+2 n}
\end{aligned}
$$

and $\Delta=R / N=r / n$.

Following the same prescription for the scattering of non-identical particles, we may define the function $Z_{N}(\ell)$ and the matrix $\mathfrak{R}(\ell)$ as follows : 


$$
\begin{aligned}
& Z_{N}(\ell)=\frac{N}{2}\left(\frac{\varphi_{N+1}-\varphi_{N-1}}{\varphi_{N}}\right) \\
& \mathfrak{R}(\ell)=\left.\frac{\psi_{\ell}}{\psi_{\ell}^{\prime}}\right|_{r=R}=R\left[\ell / 2+1+R\left(\varphi_{\ell}^{\prime} / \varphi_{\ell}\right)\right]^{-1}
\end{aligned}
$$

where $\psi_{\ell}$ is given by (8). Using the central difference approximation for the first derivative, one can relate $Z_{N}(\ell)$ to $\mathfrak{R}(\ell)$ through the following relation:

$$
Z_{N}(\ell) \cong \frac{R}{\mathfrak{R}(\ell)}-\frac{\ell}{2}-1
$$

which holds in the first approximation. As pointed out by Alam [27], the cutoff radius $R$ is chosen for a comparatively larger value, $R=5.4 \mathrm{fm}$, with also maintaining $\Delta^{2} / R$ less than unity in order to minimize the effect of the next correction term. The consideration of this correction term requires the modification of the code [28]. In addition, it is worth to mention that the phase shift analyses contain errors that propagate and accumulate during the inverse process. As such, the effect of these errors are more pronounced near the origin, i. e. for small values of $r$. These errors can be reduced by having a small number of contributing partial waves, i. e. by reducing the number of steps in the inverse process. Luckily, it is the case here as there is a reasonable number of partial waves. All this positively contribute to the performance of IST in obtaining more accurate potential points. As such, a parameterized potential close to these inverted potential points and successful in explaining the measured cross sections is very confident. 
For the scattering of two identical particles, one can calculate $Z_{N}(\ell)$ for all even partial waves $\ell=0,2,4, \ldots, L$ where $N=L / 2+1$. Also for $n=N$, or equivalently $r=R$, the potential at the surface is known, $U_{N}=(Z e)^{2} / R$, and one can easily use (23) to calculate $A_{N}(\ell)$ for all available even partial waves. In addition, $B_{N}(\ell)$ and $C_{N}(\ell)$ can be calculated using (24) and (25).

Now equation (22) can be rewritten, with $n=N$, as

$$
\frac{\varphi_{N}}{\varphi_{N-1}}=\left[C_{N}(\ell)-1\right] /\left[\frac{2}{N} Z_{N}(\ell)-A_{N}(\ell) B_{N}(\ell)\right]
$$

Defining

$$
\ell_{n}=2(N-n-1) \quad n=1,2, \ldots, N-1
$$

Eq. (25) gives $C_{n}\left(\ell_{N-n}\right)=0$; and from equation (22) we get

$$
A_{N-1}\left(\ell_{1}\right)=\frac{\varphi_{N}\left(\ell_{1}\right)}{\varphi_{N-1}\left(\ell_{1}\right)} \frac{1}{B_{N-1}\left(\ell_{1}\right)}
$$

This process can be continued inward to find all $A_{N-j}$ for $j=2,3, \ldots, N-1$ from the following general formula :

$$
A_{N-j}\left(\ell_{j}\right)=\frac{1}{B_{N-j}\left(\ell_{j}\right)}\left[\begin{array}{l}
\frac{C_{N+1-j}\left(\ell_{j}\right)}{-A_{N+1-j}\left(\ell_{j}\right) B_{N+1-j}\left(\ell_{j}\right)+\ldots}+ \\
\frac{C_{N-2}\left(\ell_{j}\right)}{-A_{N-2}\left(\ell_{j}\right) B_{N-2}\left(\ell_{j}\right)+}-A_{N-1}\left(\ell_{j}\right) B_{N-1}\left(\ell_{j}\right)+\varphi_{N}\left(\ell_{j}\right) / \varphi_{N-1}\left(\ell_{j}\right)
\end{array}\right]
$$

Then the determination of $U_{N-j}$ and $V_{N-j}$ potential points follow from (23) and (5), respectively, 


$$
\begin{aligned}
& U_{N-j}=\frac{1}{\Delta^{2}}\left[A_{N-j}\left(\ell_{j}\right)-2+\Delta^{2} k^{2}-\frac{\ell_{j}\left(3 \ell_{j}+2\right)}{4(N-j)^{2}}\right] \\
& V_{N-j}=\frac{\hbar^{2}}{2 \mu} U_{N-j}
\end{aligned}
$$

These inverted potential points are used as a constraint in determining the best parameterized potential $V(r)$, given in (1), by using a suitable fitting method for the measured angular distributions.

\section{Results and discussion}

Benefiting from the available phase shifts and the use of IST in extracting the potential points, the analytical form of the $\alpha-\alpha$ optical potential has been parameterized to remain as close as possible to the inverted potential points and, simultaneously to provide the best fit to the experimental angular distributions for each of the cases which are under consideration. The parameters of the potential that fulfil both, a good match with the inverted potential points, and a good fit to the measured angular distributions for all energies considered herein, are tabulated in Table 1. It is of great importance to discuss our results, and to compare our calculations to other available ones.

In Figs. 1 and 2, the left side compares the real potential points, obtained from inverse scattering method, with the analytical forms of the parameterized real potentials, given in (1), used in calculating the differential and reaction cross sections at all seven energies under consideration. The agreement is reasonable especially for $r \geq 2 f m$ and starts deteriorating little at $r<2 f m$. This copes with our results [2026], noting that both the less number of partial waves and the suitable choice of cutoff radius contribute to a better accuracy in the obtained inverted potential points, especially to the origin. The mild kink that appears at $r<1 \mathrm{fm}$ in the shape of the real potential has attracted 
attention. Farid [2] recommended further theoretical investigations in an attempt to know its physical origin, while Rao et al. [4] have attributed it to a sudden onset of the short-range component of the nuclear interaction or, more clearly, to the interplay between the attractive and repulsive real parts. Evidently, it is explained by our parameterized potential as obviously pronounced at 63.9 , 69.9 and 77.6 MeV.

At the energies $58.5,63.9,69.9$ and $77.6 \mathrm{MeV}$, the moderate tops and bottoms appearing in the differential cross sections in the angular range $30-60^{\circ}$ are of special concern. It was almost impossible to improve the fit and account, reasonably, for these data without the inclusion of the short-range repulsive core. As such, our results support the addition of a repulsive core to the attractive real potential term. For all these energies, the agreement between the calculated and observed differential cross sections is reasonably good. To assess the quality of such an agreement, knowing that no error bars are extracted from the experimental points, we have assumed a uniform error bar of $\pm 5 \%$ in calculating the chi-square, $\chi^{2}$, value for each fit using the well-known definition:

$$
\chi^{2}=\frac{1}{N} \sum_{i=1}^{N}\left[\frac{\left(\frac{d \sigma}{d \Omega}\right)_{\exp t}\left(\theta_{i}\right)-\left(\frac{d \sigma}{d \Omega}\right)_{\text {theor }}\left(\theta_{i}\right)}{\left(\Delta \frac{d \sigma}{d \Omega}\right)_{\exp t}\left(\theta_{i}\right)}\right]^{2}
$$

where $\left(\frac{d \sigma}{d \Omega}\right)_{\exp t}\left(\theta_{i}\right)$ and $\left(\Delta \frac{d \sigma}{d \Omega}\right)_{\exp t}\left(\theta_{i}\right)$ are, respectively, the experimental differential cross section and the corresponding error at the scattering angle $\theta_{i}$; and $\left(\frac{d \sigma}{d \Omega}\right)_{\exp t}\left(\theta_{i}\right)$ is the calculated differential cross section generated from the optical potential. The calculated $\chi^{2}-$ values, noted in Table 1, are greater than we expect, but still reasonable as we have fixed the parameters of the imaginary part and allowed only a marginal change in the parameters of the real part. 
At the higher two energies 99.6 and $119.9 \mathrm{MeV}$, although our calculated differential cross sections are in a good agreement with the observed and the calculated ones [2, 3], our calculated reaction cross sections differ by almost a factor of 2 or more. In fact, our calculated reaction cross sections at 99.6 and $119.9 \mathrm{MeV}$ are, respectively, 777 and $706 \mathrm{mb}$. These values are in a better agreement with the values reported by both Darriulat et al. [8] and Brown and Tang [9] compared to the ones given in refs. 2 and 3. Nevertheless, our calculated reaction cross sections at the other five energies 53.4, 58.49, 63.91, 69.91 and $77.55 \mathrm{MeV}$ (lab) are, respectively, 598, $813,884,846$ and 804 mb. These values are shown in Table 1, along with all other measured and calculated ones. One may also notice that the values of the reaction cross sections are very close to each other and they fall in the range of $790 \pm 100 \mathrm{mb}$ which may justify keeping the parameters of the imaginary part of the potential unchanged. In this regard, and for the scattering of two identical particles, it is worth mentioning that the detector which detects all projectiles scattered at angle $\theta$ also detects the recoiled targets when the projectiles are scattered by an angle $(\pi-\theta)$. This is approved in (12) and, in essence, doubles the reaction cross section and may substitute for the low reaction cross sections reported in refs. 2 and 3 at 100 and $120 \mathrm{MeV}$.

At least for the energies under consideration, the real potential parameters listed in Table 1 clearly show that one can consider the parameters for the first five energies as one group and the other parameters as another group. This means that one can fit the observed angular distributions by using only two sets of parameters. This is also supported by the behavior of the observed differential cross sections, especially between $30^{\circ}$ and $60^{\circ}$, where it shows a deeper slope at 99.6 and $119.9 \mathrm{MeV}$ compared to the other lower five energies. Nevertheless, this is also reflected in the general trend of the potential points extracted from the available phase shifts using IST. As such, no wonder then that no one has tried to analyze the observed angular distributions at the lower five energies: 53.4, 58.5, 63.9, 69.9 and 77.6 $\mathrm{MeV}$. 
Table 1. The real potential parameters $V_{o}($ in $\mathrm{MeV}), R_{o}($ in $\mathrm{fm}), a_{o}($ in $\mathrm{fm}), V_{1}($ in $\mathrm{MeV}), R_{1}($ in $f m)$ and $a_{1}($ in $f m)$ used in (1) for incident $\alpha$-particles with energies $E_{\alpha}($ in $\mathrm{MeV})$, in lab. system, noted in column one and incident on helium-4 target. Our calculated reaction cross sections $\sigma_{r}$ (theor) in millibarns, noticed in column eight, are compared to other published values, in indicated references, reported in columns 9-12. In column 13, $J_{R}\left(\right.$ in $\left.M e V . f m^{3}\right)$, shows the calculated real potential volume integrals per nucleon pair. The last column, $\chi^{2}$, summarizes our calculated values of chi-square per point for the fits. The parameters of the imaginary part of the potential given in (1) are kept fixed as $4 W_{s}=-100.0 \mathrm{MeV}, R_{i}=3.20 \mathrm{fm}$ and $a_{i}=0.10 \mathrm{fm}$ with a corresponding imaginary potential volume integral per nucleon pair $\left(J_{I}=80.6 \mathrm{MeV} \cdot \mathrm{fm}^{3}\right)$.

\begin{tabular}{ccccccccccccccc}
\hline$E_{\alpha}$ & $V_{o}$ & $R_{o}$ & $a_{o}$ & $V_{1}$ & $R_{1}$ & $a_{1}$ & $\sigma_{r}$ (theor $)$ & $\sigma_{r}($ Ref.8 $)$ & $\sigma_{r}($ Ref.9) & $\sigma_{r}($ Ref.2) & $\sigma_{r}($ Ref.3) & $J_{R}$ & $\chi^{2}$ \\
\hline 53.4 & -176 & 2.282 & 1.25 & 25 & 0.90 & 0.15 & 598 & 649.9 & 569 & - & - & 546.4 & 10.2 \\
58.5 & -176 & 2.357 & 1.60 & 50 & 0.68 & 0.15 & 813 & 687.9 & - & - & - & 751.3 & 40.6 \\
63.9 & -176 & 2.457 & 1.55 & 55 & 0.90 & 0.15 & 884 & 800.2 & 859 & - & - & 779.8 & 27.2 \\
69.9 & -174 & 2.377 & 1.50 & 55 & 1.00 & 0.15 & 846 & 859.3 & - & - & - & 694.5 & 48.9 \\
77.6 & -147 & 2.557 & 1.30 & 35 & 1.00 & 0.10 & 804 & 848.1 & 902 & - & - & 599.8 & 28.4 \\
99.6 & -164 & 2.177 & 1.40 & 20 & 0.30 & 0.10 & 777 & 791.5 & 868 & 387 & 350 & 521.0 & 20.4 \\
119.9 & -164 & 1.997 & 1.33 & 10 & 0.30 & 0.10 & 706 & 823.9 & 823 & 400 & 323 & 418.1 & 54.9 \\
\hline
\end{tabular}



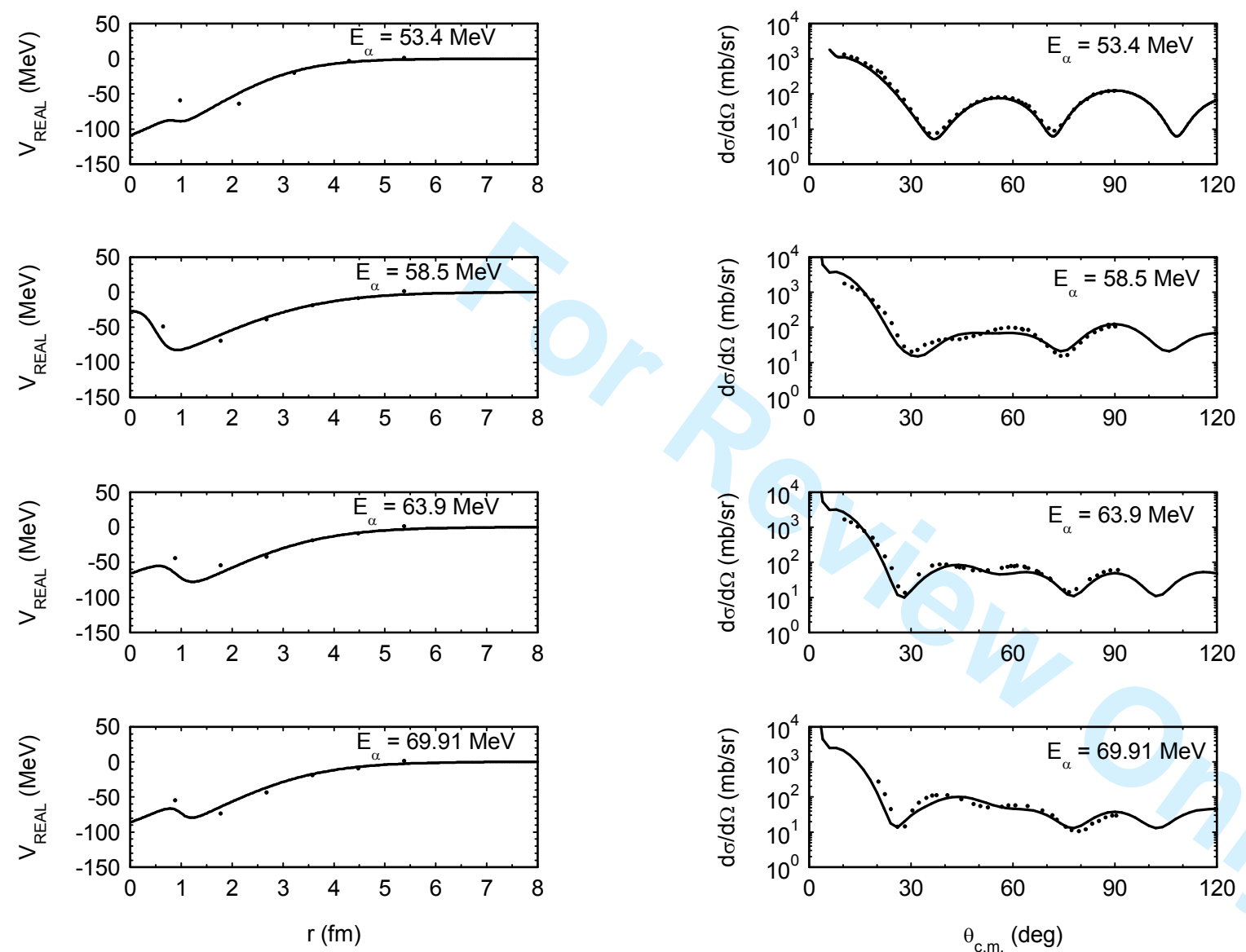

Fig. 1. The left side indicates the parameterized analytical forms of the real parts of our potentials given in Eq. (1) compared to the extracted potential points obtained from the IST. The right side shows the good agreement between calculated and measured elastic differential cross sections [8] for $\alpha-\alpha$ system at the four indicated incident energies: 53.4, 58.5, 63.9 and 69.91 MeV (lab). 

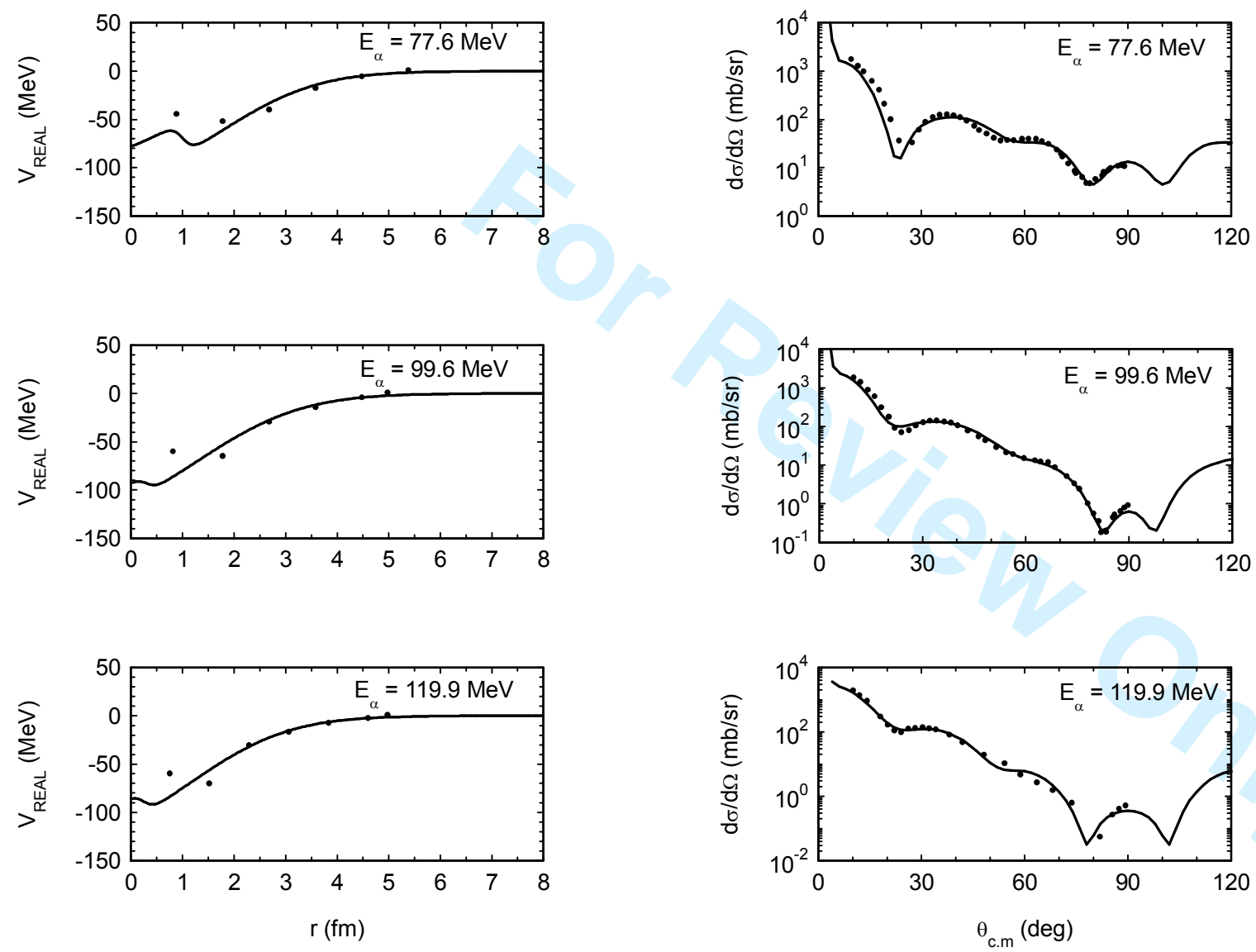

Fig. 2. Same as Fig. 1 but for the three indicated incident energies: 77.6, 99.6 and $119.9 \mathrm{MeV}$. 
Moreover, the real and imaginary volume integrals per nucleon pair, $J_{R}$ and $J_{I}$, respectively, given by [4] are also used to characterize the potential $V(r)$. Since the imaginary parameters are kept unchanged, the calculated $J_{I}$ is constant and equals 80.6 MeV. $\mathrm{fm}^{3}$. This value is almost the average of the values obtained by Rao et al. [4] and Nadasen et al. [5] at all energies under consideration. The calculated values of $J_{R}$, noted in Table 1, didn't show a systematic trend for the energy dependence as noted by others $[2,4,5]$, but they are in accord with the calculated reaction cross sections as clearly represented in Fig. 3 . This is physically expected since $J_{I}$ is energy-independent. 


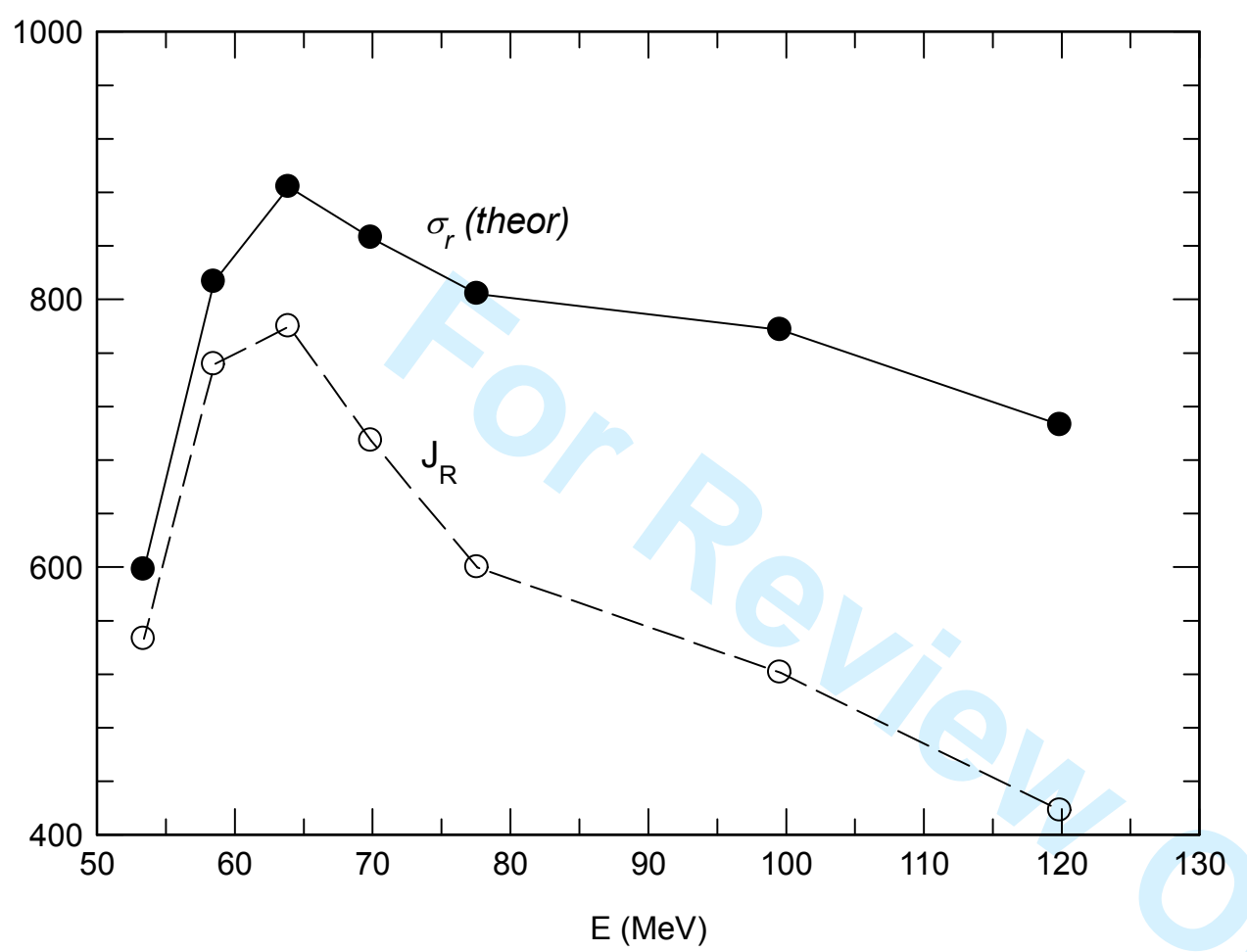

Fig. 3. The calculated reaction cross sections and real volume integrals versus energy. The solid and dashed lines are just to guide the eye. 
Since the best-fit optical model results should include not only the best fit to the elastic scattering cross sections but also a good fit to the real and imaginary phase shifts of ref. [8], we have calculated the real and imaginary parts of the phase shifts using our parameterized potential for all cases. Here we have tabulated our calculated phase shifts for only one case; namely the 99.6 incident energy, and compared them with the ones reported by Darriulat et al. [8] as indicated in Table 2. The agreement is reasonably good; and all other cases are of the same quality.

Table 2. Our calculated real and imaginary parts of the phase shifts, $\operatorname{Re} \delta_{\ell}$ and $\operatorname{Im} \delta_{\ell}$, are compared with the empirical values of their counterparts deduced from Darriulat et al.'s analysis for $E_{\alpha}=99.60 \mathrm{MeV}$.

\begin{tabular}{|c|c|c|c|c|}
\hline \multicolumn{5}{|c|}{$E_{\alpha}=99.60 \mathrm{MeV}$} \\
\hline$\ell$ & $\operatorname{Re} \delta_{\ell}$ & $\operatorname{Re} \delta_{\ell}$ (Darriulat et al.'s) & $\operatorname{Im} \delta_{\ell}$ & $\operatorname{Im} \delta_{\ell}$ (Darriulat et al.'s) \\
\hline 0 & -135.6 & $-126.0--140.7$ & 11.1 & $10.8-27.4$ \\
\hline 2 & -16.6 & $-2.0--6.40$ & 13.4 & $13.2-17.0$ \\
\hline 4 & 148.5 & $128.0-133.6$ & 20.5 & $11.2-20.9$ \\
\hline 6 & 85.9 & $86.7-90.0$ & 33.9 & $27.0-30.3$ \\
\hline 8 & 19.6 & $16.9-21.5$ & 7.5 & $8.5-15.5$ \\
\hline 10 & 10.0 & $4.3-6.8$ & 0.2 & $0.0-1.0$ \\
\hline
\end{tabular}




\section{Conclusions}

Our new simple local optical potential, which is different from all reported potentials, proves to be successful in providing a reasonable simultaneous explanation for the observed differential and reaction cross sections at the seven laboratory bombarding energies: $E_{\alpha}=53.4,58.5,63.9,69.9,77.6,99.6$, and 119.9MeV . This investigation represents the first endeavor to analyze the data at the lower five energies. The results of the IST have been used as constraints in determining the potential parameters and, as such, the correct potentials.

Our $\alpha-\alpha$ real potential has a short-range repulsive core with a longer range attraction, not a sum of two attractive potentials. This helps in explaining the observed kink in the real potential at $r<1 \mathrm{fm}$; and in improving the fit for the differential angular distribution data in the angular range $30^{\circ}-60^{\circ}$. Moreover, our calculated reaction cross sections are in a good agreement with the ones reported in refs. 8 and 9. In addition, our calculated phase shifts are comparable with the ones reported in ref. 8 . Hence, this new potential might be considered as a remarkable success that one can build on to determine alpha-alpha potentials, in other energy regions, and also alpha-nucleus potentials. 


\section{Acknowledgements}

The author acknowledges the financial support of the Deanship of Scientific Research at Taif University, Taif, Saudi Arabia for carrying out this investigation. My special thanks go to the referee and associate editors for their valuable comments and suggestions. This paper is dedicated to the soul of Prof. F. B. Malik who passed away when this work was in its final stages.

\section{References}

1. M. N. A. Abdullah, M. S. Sabra, M. M. Rashid, Z. F. Shehadeh, M. M. Billah, S. K. Das, M. A.

Uddin, A. K. Basak, I. Reichstein, H. M. Sen Gupta, and F. B. Malik. Nucl. Phys. A, 775, 1 (2006).

2. M. El-Azab Farid, Phys. Rev. C, 74, 064616-1 (2006).

3. D. Chauhan and Z. A. Khan. Eur. Phys. J. A, 41, 179 (2009).

4. K. A. G. Rao, A. Nadasen, D. Sisan, W. Yuhasz, D. Mercer, S. M. Austin, P. G. Ross, and R. E. Warner. Phys. Rev. C, 62, 014607-1 (2000).

5. A. Nadasen, P. G. Ross, B. G. Galgola, G. J. Mathews, V. E. Viola, Jr., H. G. Pugh, and P. Frisbee. Phys. Rev. C, 18, 2792 (1978).

6. W. R. Coker and T. Tamura. Phys. Lett. B, 62, 374 (1976).

7. J. C. Fong, M. M. Gazzaly, G. Igo, A. D. Liberman, R. J. Ridge, S. L. Verbeck, C. A. Whitten, V. Perez-Mendez, and W. R. Coker. Nucl. Phys. A, 262, 365 (1976).

8. P. Darriulat, G. Igo, H. G. Pugh, and H. D. Holmgren. Phys. Rev. 137, B315 (1965).

9. R. E. Brown and Y. C. Tang. Nucl. Phys. A, 170, 225 (1971).

10. F. C. Barker. Aust. J. Phys. 22, 293 (1969). 
11. V. G. Neudatchin, V. I. Kukulin, V. L. Korotkikh, and V. P. Korennoy. Phys. Lett. B, 34, 581 (1971).

12. W. Czyz and L. C. Maximon. Ann. Phys. (N.Y.) 52, 59 (1969).

13. R. J. Glauber. In Proceedings of the $2^{\text {nd }}$ International Conference on High Energy Physics and Nuclear Structure, vol. 1. Edited by G. Alexander. North-Holland, Amsterdam. 1967. p. 311.

14. P. Swan. Ann. Phys. (N.Y.) 48, 455 (1968).

15. G. F. Steyn, S. V. Fortsch, J. J. Lawrie, F. D. Smit, R. T. Newman, A. A. Cowley, and R. Lindsay. Phys. Rev. C, 54, 2482 (1996).

16. G. F. Steyn, S. V. Fortsch, A. A. Cowley, S. Karataglidis, R. Lindsay, J. J. Lawrie, F. D. Smit, and R. T. Newman. Phys Rev. C, 57, 1817 (1998).

17. A. K. Jain and B. N. Joshi. Prog. Theor. Phys. 120, 1193 (2008).

18. M. M. Alam and F. B. Malik. Phys. Lett. B, 237, 14 (1990).

19. M. M. Alam and F. B. Malik. Nucl. Phys. A, 524, 88 (1991).

20. Z. F. Shehadeh. Int. Jour. Mod. Phys. E, 18, 1615 (2009).

21. Z. F. Shehadeh, J. S. Scott, and F. B. Malik. American Institute of Physics Journal 1370, 185 (2011).

22. Z. F. Shehadeh. JAAUBAS 14, 32 (2013).

23. Z. F. Shehadeh. Turk. J. Phys. 37, 190 (2013).

24. Z. F. Shehadeh. J. Mod. Phys. 5, 341 (2014).

25. Z. F. Shehadeh. Int. Rev. Phys. 8, 110 (2014).

26. Z. F. Shehadeh. J. Mod. Phys. 5, 1652 (2014).

27. M. M. Alam. Ph. D. Thesis, Southern Illinois University at Carbondale, Illinois, USA (1991).

28. M. M. Alam and F. B. Malik. Private communications. 

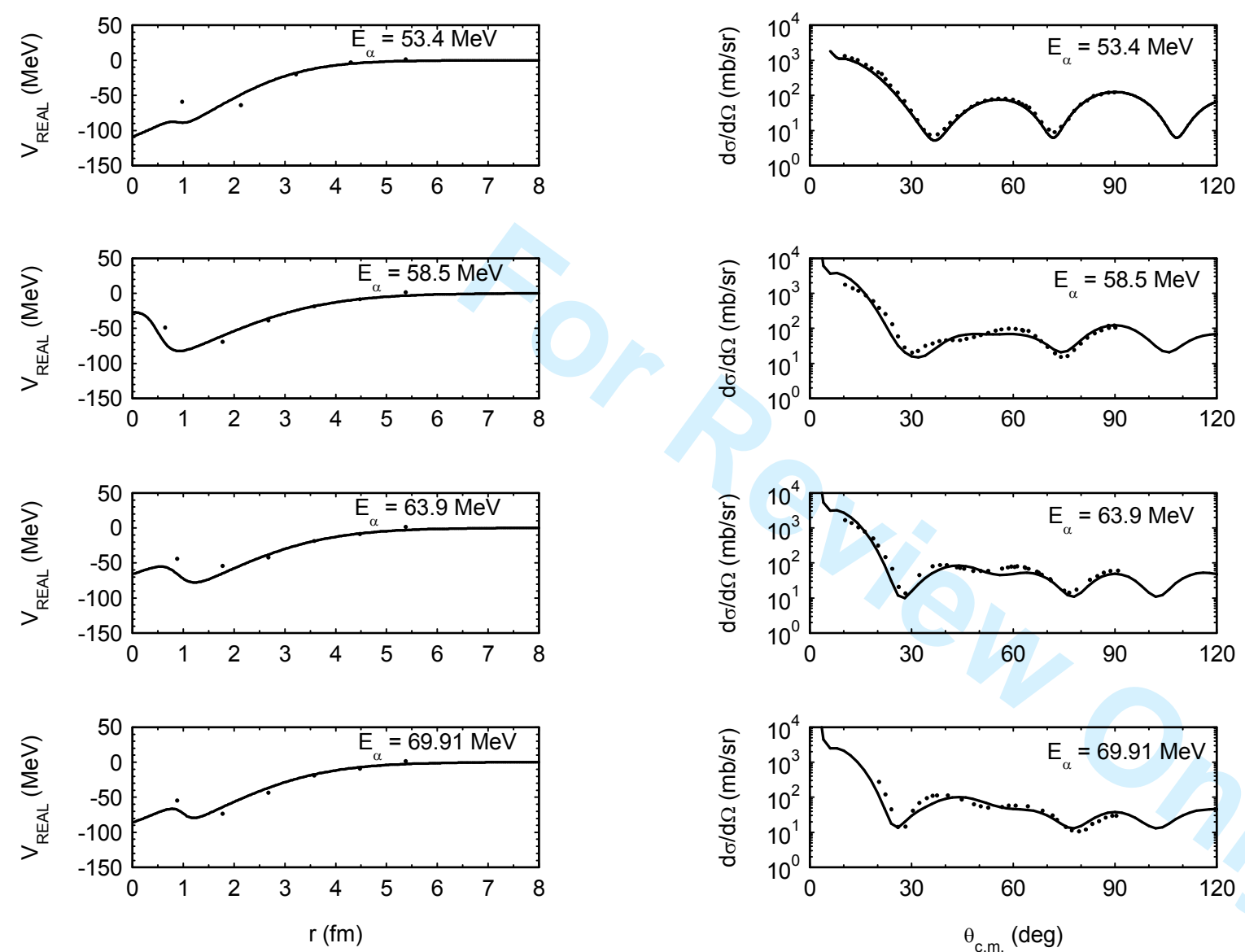

Fig. 1. The left side indicates the parameterized analytical forms of the real parts of our potentials given in Eq. (1) compared to the extracted potential points obtained from the IST. The right side shows the good agreement between calculated and measured elastic differential cross sections [8] for $\alpha-\alpha$ system at the four indicated incident energies: 53.4, 58.5, 63.9 and 69.91 MeV (lab). 

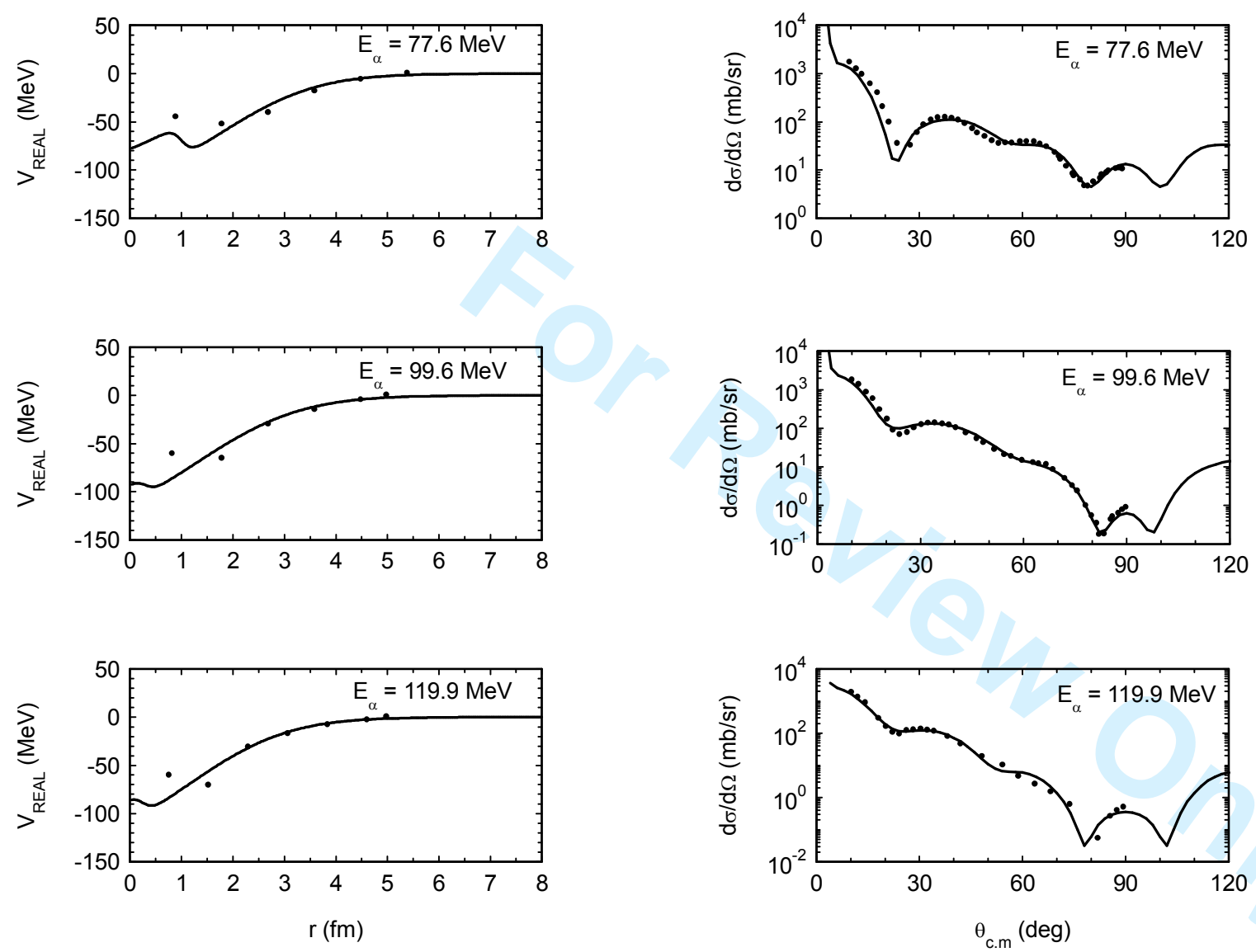

Fig. 2. Same as Fig. 1 but for the three indicated incident energies: 77.6, 99.6 and $119.9 \mathrm{MeV}$. 


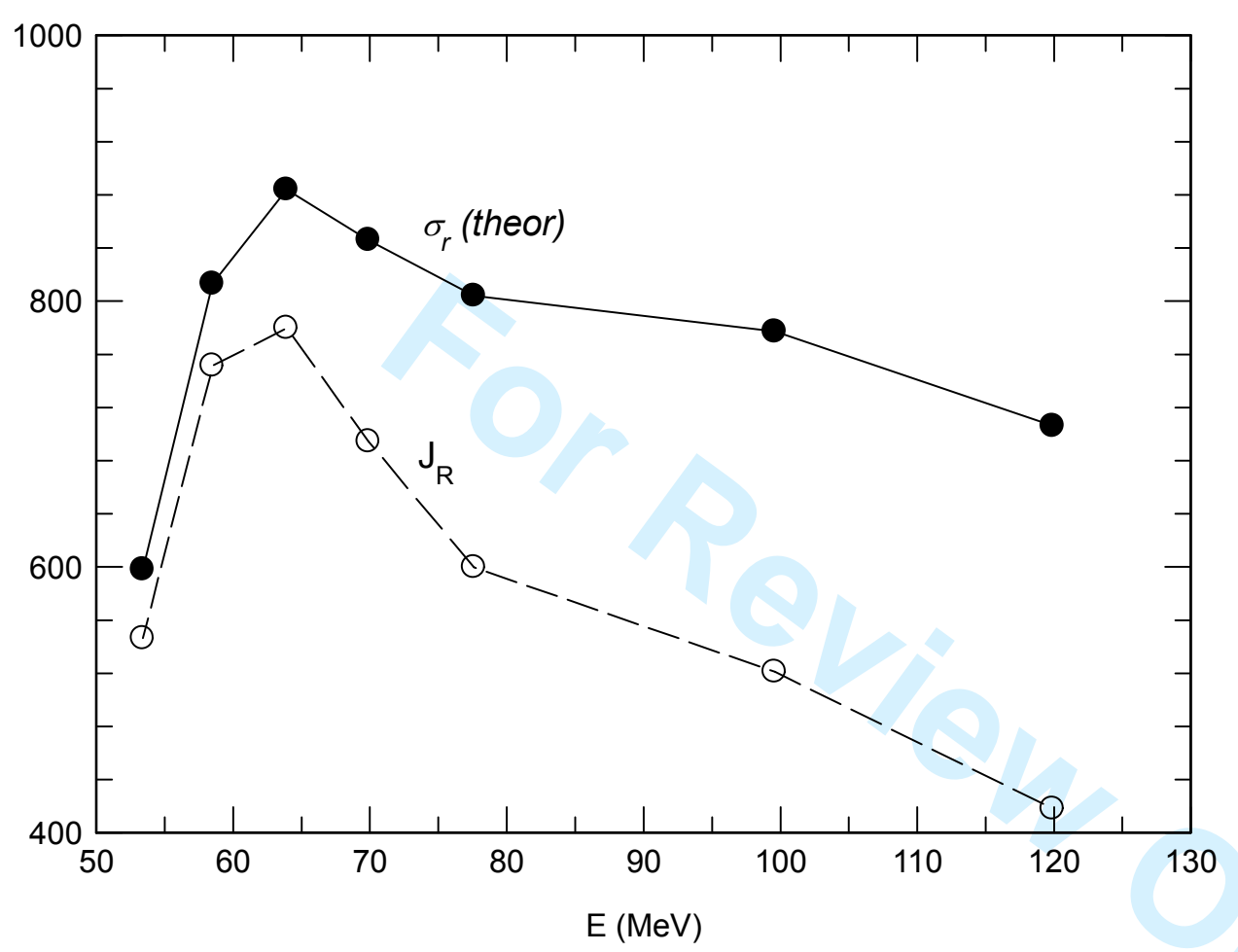

Fig. 3. The calculated reaction cross sections and real volume integrals versus energy. The solid and dashed lines are just to guide the eye. 
Table 1. The real potential parameters $V_{o}($ in $\mathrm{MeV}), R_{o}($ in $\mathrm{fm}), a_{o}($ in $\mathrm{fm}), V_{1}($ in $\mathrm{MeV}), R_{1}($ in $f m)$ and $a_{1}($ in $f m)$ used in (1) for incident $\alpha$-particles with energies $E_{\alpha}($ in $\mathrm{MeV})$, in lab. system, noted in column one and incident on helium-4 target. Our calculated reaction cross sections $\sigma_{r}$ (theor) in millibarns, noticed in column eight, are compared to other published values, in indicated references, reported in columns 9-12. In column 13, $J_{R}\left(\right.$ in $\left.M e V . f m^{3}\right)$, shows the calculated real potential volume integrals per nucleon pair. The last column, $\chi^{2}$, summarizes our calculated values of chi-square per point for the fits. The parameters of the imaginary part of the potential given in (1) are kept fixed as $4 W_{s}=-100.0 \mathrm{MeV}, R_{i}=3.20 \mathrm{fm}$ and $a_{i}=0.10 \mathrm{fm}$ with a corresponding imaginary potential volume integral per nucleon pair $\left(J_{I}=80.6 \mathrm{MeV} \cdot \mathrm{fm}^{3}\right)$.

\begin{tabular}{cccccccccccccccc}
\hline$E_{\alpha}$ & $V_{o}$ & $R_{o}$ & $a_{o}$ & $V_{1}$ & $R_{1}$ & $a_{1}$ & $\sigma_{r}$ (theor $)$ & $\sigma_{r}($ Ref.8 $)$ & $\sigma_{r}($ Ref.9) & $\sigma_{r}($ Ref.2) & $\sigma_{r}($ Ref.3) & $J_{R}$ & $\chi^{2}$ \\
\hline 53.4 & -176 & 2.282 & 1.25 & 25 & 0.90 & 0.15 & 598 & 649.9 & 569 & - & - & 546.4 & 10.2 \\
58.5 & -176 & 2.357 & 1.60 & 50 & 0.68 & 0.15 & 813 & 687.9 & - & - & - & 751.3 & 40.6 \\
63.9 & -176 & 2.457 & 1.55 & 55 & 0.90 & 0.15 & 884 & 800.2 & 859 & - & - & 779.8 & 27.2 \\
69.9 & -174 & 2.377 & 1.50 & 55 & 1.00 & 0.15 & 846 & 859.3 & - & - & - & 694.5 & 48.9 \\
77.6 & -147 & 2.557 & 1.30 & 35 & 1.00 & 0.10 & 804 & 848.1 & 902 & - & - & 599.8 & 28.4 \\
99.6 & -164 & 2.177 & 1.40 & 20 & 0.30 & 0.10 & 777 & 791.5 & 868 & 387 & 350 & 521.0 & 20.4 \\
119.9 & -164 & 1.997 & 1.33 & 10 & 0.30 & 0.10 & 706 & 823.9 & 823 & 400 & 323 & 418.1 & 54.9 \\
\hline
\end{tabular}


Table 2. Our calculated real and imaginary parts of the phase shifts, $\operatorname{Re} \delta_{\ell}$ and $\operatorname{Im} \delta_{\ell}$, are compared with the empirical values of their counterparts deduced from Darriulat et al.'s analysis for $E_{\alpha}=99.60 \mathrm{MeV}$.

\begin{tabular}{|c|c|c|c|c|}
\hline \multicolumn{5}{|c|}{$E_{\alpha}=99.60 \mathrm{MeV}$} \\
\hline$\ell$ & $\operatorname{Re} \delta_{\ell}$ & $\operatorname{Re} \delta_{\ell}$ (Darriulat et al.'s) & $\operatorname{Im} \delta_{\ell}$ & $\operatorname{Im} \delta_{\ell}$ (Darriulat et al.'s) \\
\hline 0 & -135.6 & $-126.0--140.7$ & 11.1 & $10.8-27.4$ \\
\hline 2 & -16.6 & $-2.0--6.40$ & 13.4 & $13.2-17.0$ \\
\hline 4 & 148.5 & $128.0-133.6$ & 20.5 & $11.2-20.9$ \\
\hline 6 & 85.9 & $86.7-90.0$ & 33.9 & $27.0-30.3$ \\
\hline 8 & 19.6 & $16.9-21.5$ & 7.5 & $8.5-15.5$ \\
\hline 10 & 10.0 & $4.3-6.8$ & 0.2 & $0.0-1.0$ \\
\hline
\end{tabular}

